\title{
NLRP7 plays a functional role in regulating BMP4 signaling during differentiation of patient-derived trophoblasts
}

\author{
Aybuke Alici-Garipcan (1)', Burcu Özçimen², Ilke Süder', Volkan Ülker ${ }^{3}$ Tamer T. Önder ${ }^{2}$ and Nesrin Özören ${ }^{1}$
}

\begin{abstract}
Complete hydatidiform mole (HM) is a gestational trophoblastic disease resulting in hyperproliferation of trophoblast cells and absence of embryo development. Mutations in the maternal-effect gene NLRP7 are the major cause of familial recurrent complete HM. Here, we established an in vitro model of HM using patient-specific induced pluripotent stem cells (iPSCs) derived trophoblasts harboring NLRP7 mutations. Using whole transcriptome profiling during trophoblast differentiation, we showed that impaired NLRP7 expression results in precocious downregulation of pluripotency factors, activation of trophoblast lineage markers, and promotes maturation of differentiated extraembryonic cell types such as syncytiotrophoblasts. Interestingly, we found that these phenotypes are dependent on BMP4 signaling and BMP pathway inhibition corrected the excessive trophoblast differentiation of patient-derived iPSCs. Our human iPSC model of a genetic placental disease recapitulates aspects of trophoblast biology, highlights the broad utility of iPSC-derived trophoblasts for modeling human placental diseases and identifies NLRP7 as an essential modulator of key developmental cell fate regulators.
\end{abstract}

\section{Introduction}

Maternal-effect genes encode for proteins, deposited into oocytes, which are required for embryonic development ${ }^{1}$. Mutations in such genes result in embryonic phenotypes that reflect the genotype of the mother rather than that of the offspring ${ }^{2}$. In mice models, null phenotypes of a majority of these genes result in arrested development at very early embryonic time points ${ }^{3}$. Familial biparental hydatidiform mole (FBHM; MIM 231090) is the only known pure maternal-effect inherited disorder in humans. Complete molar pregnancy $(\mathrm{CHM})$ is a gestational trophoblastic disease, defined as a pregnancy with no embryo along with abnormal hyperproliferation of extraembryonic

\footnotetext{
Correspondence: Nesrin Özören (nesrin.ozoren@boun.edu.tr)

${ }^{1}$ Apoptosis and Cancer Immunology Laboratory (AKIL), Department of Molecular Biology and Genetics, Center for Life Sciences and Technologies, Bogazici University, Istanbul, Turkey

${ }^{2}$ School of Medicine, Koç University, Istanbul, Turkey

Full list of author information is available at the end of the article These authors contributed equally: Tamer T. Önder, Nesrin Özören Edited by Y. Shi
}

trophoblastic tissue ${ }^{4,5}$. The major cause for FBHM is homozygous or compound heterozygous maternal-effect mutations in NLRP7 (NOD-like receptor family pyrin domain containing 7$)^{6-9}$. Certain NLRP7 mutations found in a heterozygous state have also been associated with recurrent reproductive failure ${ }^{10}$. Interestingly, two of the first mammalian maternal-effect genes identified encode for NLR proteins, Nlrp5 (Mater) and Nlrp2, both of which are required for early embryonic development of mice and have unknown functions ${ }^{11,12}$. In contrast to mice models of maternal-effect genes, embryos of affected women with NLRP7 mutations do not arrest at a very early stage, but rather undergo excessive differentiation and commitment to extraembryonic lineages in vivo.

The NLRP7 gene is absent in rodents ${ }^{13,14}$. NLRP7 has been reported to be involved in inflammasome formation and cytokine release $\mathrm{e}^{15,16}$ as well as in modulating DNA methylation and trophoblast differentiation ${ }^{17,18}$. FBHM associated NLRP7 mutations have been linked to imprinting abnormalities $^{19}$. However, how NLRP7 regulates early embryonic

\section{(c) The Author(s) 2020}

(c) (i) Open Access This article is licensed under a Creative Commons Attribution 4.0 International License, which permits use, sharing, adaptation, distribution and reproduction cc) in any medium or format, as long as you give appropriate credit to the original author(s) and the source, provide a link to the Creative Commons license, and indicate if changes were made. The images or other third party material in this article are included in the article's Creative Commons license, unless indicated otherwise in a credit line to the material. If material is not included in the article's Creative Commons license and your intended use is not permitted by statutory regulation or exceeds the permitted use, you will need to obtain permission directly from the copyright holder. To view a copy of this license, visit http://creativecommons.org/licenses/by/4.0/. 
development and how its absence contributes to human disease pathogenesis remain unclear. As mice lack NLRP7, it has not been possible to study the role of this maternal-effect gene during embryogenesis in model organisms.

Patient-derived disease-specific induced pluripotent stem cells (iPSCs) provide a powerful platform for overcoming the ethical and technical challenges associated with studying early human embryogenesis. To derive trophoblasts from hESCs several groups have reported the feasibility of BMP4 treatment, alone or in combination with inhibitors of FGF and ACTIVIN/NODAL signaling $^{20-22}$. Here, we established iPSCs-derived trophoblasts from an HM patient with impaired NLRP7 expression and showed that these cells provide a model to investigate human placental diseases. Finally, our work demonstrates that NLRP7 plays a pivotal role in trophoblast differentiation by modulating the BMP4 pathway.

\section{Results}

\section{Generation of human patient-derived iPSC from HM patient with NLRP7 mutations}

To assess the function of NLRP7 in HM pathology, we generated iPSC from a patient carrying compound heterozygous variant of NLRP7 with a prior diagnosis of recurrent $\mathrm{HM}^{23,24}$ (Fig. 1a). Fibroblasts from a $\mathrm{HM}$ patient and an unrelated healthy control (WT) were reprogrammed using non-integrating episomal plasmids $^{25,26}$. No difference was observed between HM and WT cells in terms of reprogramming efficiency and maintenance of pluripotency under standard culture conditions (Fig. 1b-d). iPSCs expressed pluripotency markers OCT4, NANOG, SOX2, LIN28A, and LIN28B as assessed by immunofluorescence and RT-PCR (Fig. 1c, d). iPSC lines were karyotypically normal, devoid of episomal reprogramming vectors, and could readily differentiate into cell types belonging to three germ layers in a teratoma formation assay in immunocompromised mice (Fig. S1a-c).

Subsequently, we confirmed severely reduced expression of NLRP7 (88\% loss of expression) in HM cells at the molecular level (Figs. 1e, f and S1d). We observed that reprogramming promoted the expression of NLRP7 in WT cells, while $\mathrm{HM}^{\mathrm{iPSC}}$ had significantly reduced expression (Fig. S1e). On the other hand, NLRP2, the closest homolog of NLRP7, levels were not affected in patient-derived iPSCs (Figs. S1d and 1e). These results indicate that somatic NLRP7 mutant cells can be successfully reprogrammed to pluripotency via exogenous transcription factors.

\section{iPSC-derived patient trophoblasts closely resemble HM pathogenesis}

Next, we generated trophoblast cells from iPSCs using an established protocol of BMP4 treatment in combination with inhibition of ALK5 and FGF signaling (via A83-01 and PD173074; referred to as BAP condition) ${ }^{22,27,28}$ (Fig. 2a). On the second day of BAP exposure, WT cells became distinguishable by their flattened morphology from undifferentiated counterparts. Intriguingly, HM cells started to lose their distinct iPSC morphology only after $24 \mathrm{~h}$ of BAP treatment (Fig. 2b).

To characterize the differentiation of iPSCs toward trophoblast-like cells at the whole transcriptome level, we carried out RNA-sequencing analysis of $\mathrm{WT}^{\mathrm{iPSC}}$ and $\mathrm{HM}^{\mathrm{iPSC}}$ over the time course of differentiation. Hierarchical clustering analysis pointed to a high degree similarity between the $\mathrm{WT}^{\mathrm{iPSC}}$ and $\mathrm{HM}^{\mathrm{iPSC}}$ indicating that NLRP7 mutations do not lead to significant transcriptional changes in the pluripotent state (Fig. S2a). Upon BAP treatment, the transcriptomes of the groups differed from their parental iPSCs. To address the extent of trophoblast differentiation in our culture system, we compiled a list of general trophoblast markers based on the recent literature (Table S1). Gradual upregulation of trophoblast genes was observed during the time course of differentiation of WT cells upon BAP exposure indicating the success of BAP to direct commitment of pluripotent stem cells to trophoblast lineage (Fig. S2b). Gene Set Enrichment Analysis (GSEA) further confirmed highly significant downregulation of ESC-specific genes and upregulation of placental genes (Fig. S2c). At the whole transcriptome level $\mathrm{HM}^{\mathrm{BAP}}$ cells were enriched in trophoblast signature whereas pluripotency signatures were attenuated in comparison to $\mathrm{WT}^{\mathrm{BAP}}$ cells in a timedependent manner (Figs. 2c-e and S2d). Of note, the transcripts of many trophoblast cell fate transcription factors were upregulated in $\mathrm{HM}^{\mathrm{BAP}}$ such as TFAP2C, GATA3, and CDX2 (Fig. 2c) $)^{29,30}$.

To further assess the differentiation efficiency, we analyzed widely used trophoblast markers: CDX2, HLA-G, KRT7 and the pluripotency marker, OCT3/4 protein expression by immunostaining. Although, BAP-treated cells of both groups started to be positive for early trophoblast lineage marker, CDX2 on day 2, HM cells tended to gain more CDX2 positivity than WT cells (Fig. 2f). On the other hand, HLA-G positive cells were visible mostly where CDX2 was absent on day 4 indicating the later stages of differentiation. Staining for HLA-G was considerably stronger and more uniform in HM cells. Although, the majority of the cells from both groups were positive for KRT7 even on day 2, HM cells stained more strongly than WT cells for all time points assessed. Consistent with the initiation of differentiation, OCT3/ 4 staining declined after BAP exposure for 2 days, with $\mathrm{HM}$ cells exhibiting a greater degree of OCT3/4 loss than WT cells. We also noticed that HM cells became enlarged and more uniform when compared to WT cells in response to BAP treatment. As increased cell size is an 
A

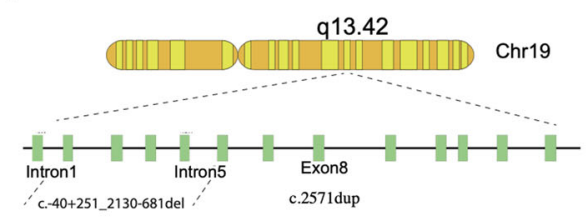

B

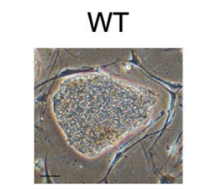

C
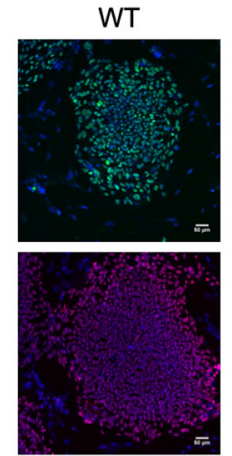

$\mathrm{HM}$

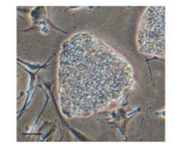

HM
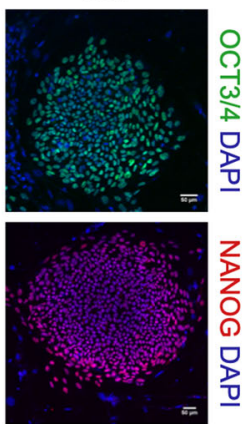

D

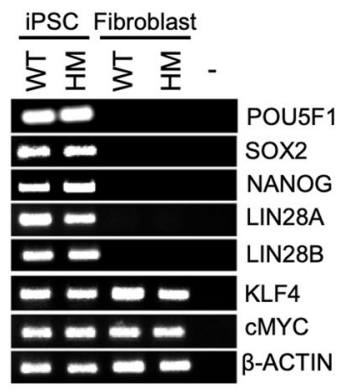

E

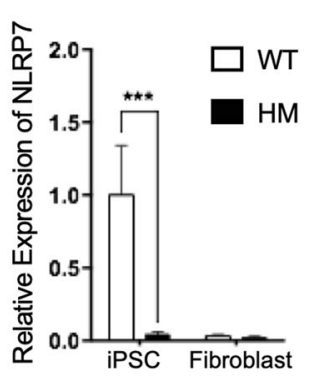

F

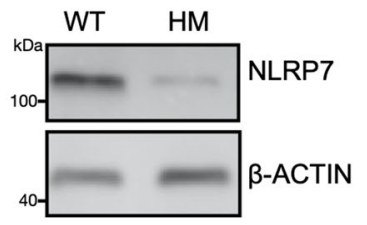

Fig. 1 Characterization of HM specific iPSCs carrying NLRP7 mutations. a Schematic and coordinates of the deletion and the single base pair duplication on NLRP7 gene in HM cells used in this study. $\mathbf{b}$ Colony morphologies of reprogrammed cells on MEFs under light microscopy. Images were acquired at $4 \mathrm{X}$ magnification. $\mathbf{c}$ Immunostaining for pluripotency markers OCT3/4 and NANOG. DAPI was used as a nuclear stain. $\mathbf{d}$ RT-PCR showing the expression of pluripotency markers in WT and HM iPSCs and their fibroblast counterparts. e NLRP7 mRNA levels in WT and HM iPSCs and their fibroblast counterparts. Relative mRNA levels were normalized to GAPDH. $n=3$ biological replicates. The bars represent mean \pm SD. ${ }^{* * *} P<0.005$ by two-way ANOVA followed by Sidak's multiple comparison test. f NLRP7 protein levels in WT and HM iPSCs as detected by immunoblotting. $\beta$-actin was used as a loading control.

initial event in trophoblastic differentiation ${ }^{31,32}$, we measured the sizes of DAPI-stained cell nuclei. HM cells had significantly larger nuclei than that of WT cells (Fig. S2e). In accordance with immunostaining results, OCT3/4 was not detectable on day 4 of BAP treatment, whereas CDX2, KRT7 and HLA-G were elevated in HM cells upon BAP exposure (Fig. 2g).

Placental growth factor (PGF) is a placental hormone produced predominantly by functional trophoblasts during pregnancy ${ }^{33}$. HM cells produced significantly more soluble PGF than WT cells on day 4 (Fig. 2h). We then tested whether BAP treatment resulted in the differentiation of a specific sub-type of trophoblasts, namely, cytotrophoblasts (CT), extravillous trophoblasts (EVT), and syncytiotrophoblasts (ST). We compiled lists of markers indicative of different trophoblast populations (Table S2) and examined their expressions during differentiation. While CT and EVT marker genes were both induced at a certain level in $\mathrm{WT}^{\mathrm{BAP}}$ and $\mathrm{HM}^{\mathrm{BAP}}$ cells, $\mathrm{ST}$ related genes such as ERVW-1 (Syncytin) were remarkably enriched in $\mathrm{HM}^{\mathrm{BAP}}$ cells in comparison to $\mathrm{WT}^{\mathrm{BAP}}$ cells. As STs are terminally differentiated trophoblasts,
HM cells appeared to exhibit a more advanced differentiation status (Fig. S2f).

Taken together, these results suggest that patientspecific iPSC-derived trophoblasts can successfully recapitulate HM disease pathogenesis and highlight an early activation of trophoblast lineage commitment dependent on impaired NLRP7 expression.

\section{BMP4 independent derivation of trophoblasts from HM iPSCs}

As BMP4 is known to induce trophoblast differentiation, we investigated whether BMP4 signaling could be responsible for the phenotype observed in HM cells. We removed BMP4 from the differentiation medium (AP) and assessed the predisposition of HM cells to differentiate to trophoblasts. Surprisingly, $\mathrm{HM}^{\mathrm{AP}}$ cells displayed predominantly homogeneous phenotype resembling the morphology of BAP-treated cells on day 4 (Fig. S3a). WT cells did not exhibit such a difference in morphology from undifferentiated iPSCs. Hierarchical clustering of whole transcriptome data demonstrated that $\mathrm{HM}^{\mathrm{AP}}$ cells were broadly distinct from $\mathrm{WT}^{\mathrm{AP}}$ cells on day 4 (Fig. S3b). 


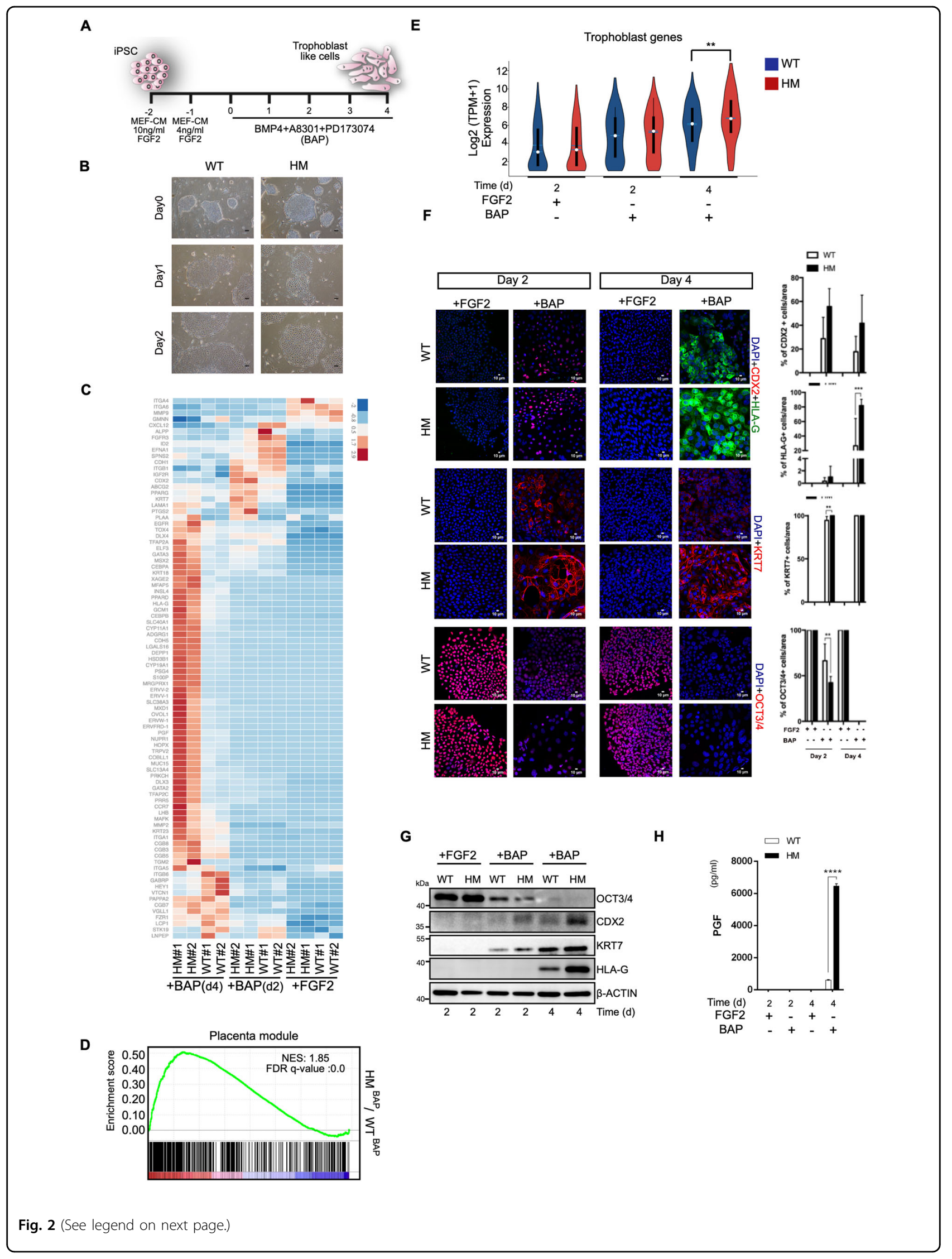




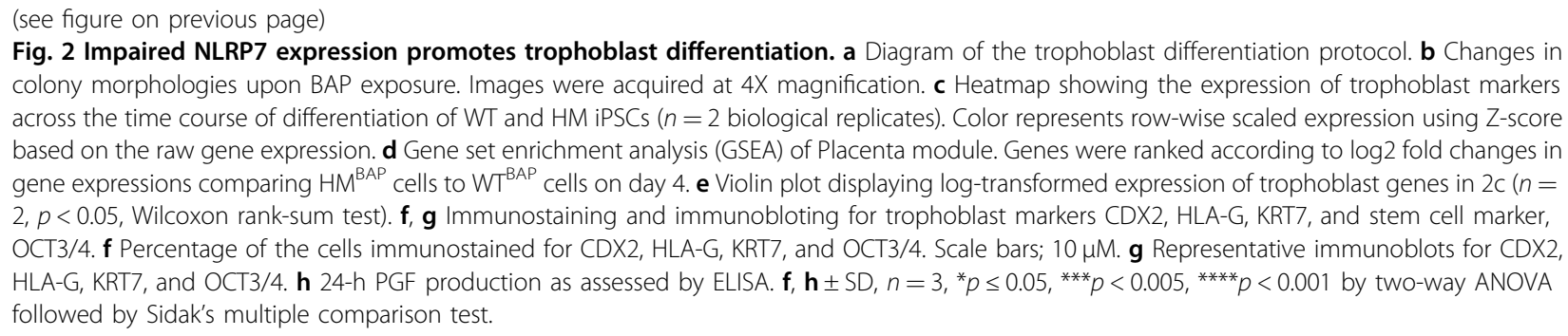

Remarkably, HM cells expressed genes related to trophoblasts to a substantial level when treated only with AP in the absence of BMP4 (Fig. 3a) and the differentially expressed transcripts in $\mathrm{HM}^{\mathrm{AP}}$ cells were highly associated with the placenta (Fig. 3b). Moreover, the global expression of trophoblast genes was enriched in $\mathrm{HM}^{\mathrm{AP}}$ cells (Fig. 3c), whereas the transcripts of pluripotency genes declined in comparison to $\mathrm{WT}^{\mathrm{AP}}$ cells (Fig. S3c). We verified that transcripts of CDX2 on day 2 and PGF, INSL4, PSG on day 4 were higher in HM cells compared to WT cells (Fig. 3d). On the other hand, no significant global upregulation was observed for the genes related to endoderm, mesoderm, or mesoendoderm differentiation (Fig. S3d).

Immunostaining revealed that CDX2 positive cells were evident in HM group on day 2 and HLA-G positive cells emerged on day 4 upon AP treatment (Fig. 3e). These results mirrored the phenotype observed for that of BAP exposure albeit at a lower level for the trophoblast specific markers. All of the HM cells strongly stained for KRT7 on day 4 unlike $\mathrm{WT}^{\mathrm{AP}}$ cells. HM cells also exhibited larger nuclei than WT cells upon AP exposure (Fig. S3e). Western blot analysis confirmed that AP-treated HM cells expressed substantially more CDX2, KRT7, and HLA-G on day 4 compared to WT cells (Fig. 3f). OCT3/4 protein levels decreased dramatically on day 4 in HM cells showing that HM cells lost pluripotency properties faster than WT cells.

PGF secretion provided a clear evidence for the trophoblastic features of $\mathrm{HM}^{\mathrm{AP}}$ cells (Fig. 3g). We next examined whether impaired NLRP7 could endow HM cells with the capacity to differentiate into terminal subtypes of trophoblasts under AP conditions. $\mathrm{HM}^{\mathrm{AP}}$ cell population contained CT, EVT, and ST-like cells as evidenced by the robust expression of trophoblast markers belonging to these subtypes on day 4 (Fig. S3f).

Since hydatidiform mole is characterized as the hyperproliferation of trophoblast cells, we evaluated cell cycle kinetics of cells. However, we could not detect enrichment for genes involved in both G1/S and G2/M transition in HM cells. Rather, we observed decreased expression of cell cycle genes on day 4 of $\mathrm{HM}^{\mathrm{BAP}}$ and
$\mathrm{HM}^{\mathrm{AP}}$ cells including well-known proliferation markers, PCNA and MKI67. This result was further confirmed via GSEA whereby "Mitosis module" was negatively enriched in $\mathrm{HM}^{\mathrm{BAP}}$ cells on day 4 compared to $\mathrm{WT}^{\mathrm{BAP}}$ cells, possibly reflecting the more advanced differentiation state of the mutant iPSC-derived cells (Fig. S4). No major significant differences in key cell cycle related genes were detected at the other time points and treatment conditions between control and HM cells suggesting that additional factors may be required to model the hyperproliferation of $\mathrm{HM}$ cells observed during pregnancy in vivo.

Next, we evaluated the differentiation kinetics of $\mathrm{HM}^{\mathrm{AP}}$ cells in comparison to BAP-treated cells. We conducted comparative analyses across treatment, time, and genotype. Notably, BAP-treated WT group, and AP-treated HM group were clustered together on day 4 pointing a broadly similar trophoblast differentiation kinetics (Fig. S5). This result further verified that trophoblast differentiation in $\mathrm{HM}^{\mathrm{AP}}$ cells does not require exogeneous addition of BMP4. Moreover, data revealed the greatest differentiation efficiency in $\mathrm{HM}^{\mathrm{BAP}}$ cells amongst all groups and thus, although exogenous BMP4 is dispensable to derive trophoblasts from HM iPSCs, it can still promote trophoblast differentiation. Overall, trophoblast differentiation of $\mathrm{HM}$ cells in the absence of exogeneous BMP4 suggested that HM cells may undergo excessive trophoblast differentiation due to dysregulation of BMP4 signaling.

\section{NLRP7 modulates BMP4 signaling during trophoblast differentiation}

Promotion of trophoblast lineage commitment without exogenous BMP4 led us to hypothesize that NLRP7 may act on trophoblast differentiation through BMP4 signaling. Interestingly, RNA-sequencing revealed that BMP signaling related genes were significantly enriched in the transcriptome of HM cells when treated with AP at the early time point (Fig. 4a). We uncovered that BMP4 mRNA levels and soluble BMP4 secretion were higher in $\mathrm{HM}^{\mathrm{AP}}$ cultures in comparison to $\mathrm{WT}^{\mathrm{AP}}$ cultures (Fig. 4b, c). As aberrant DNA methylations have been observed in recurrent familial 


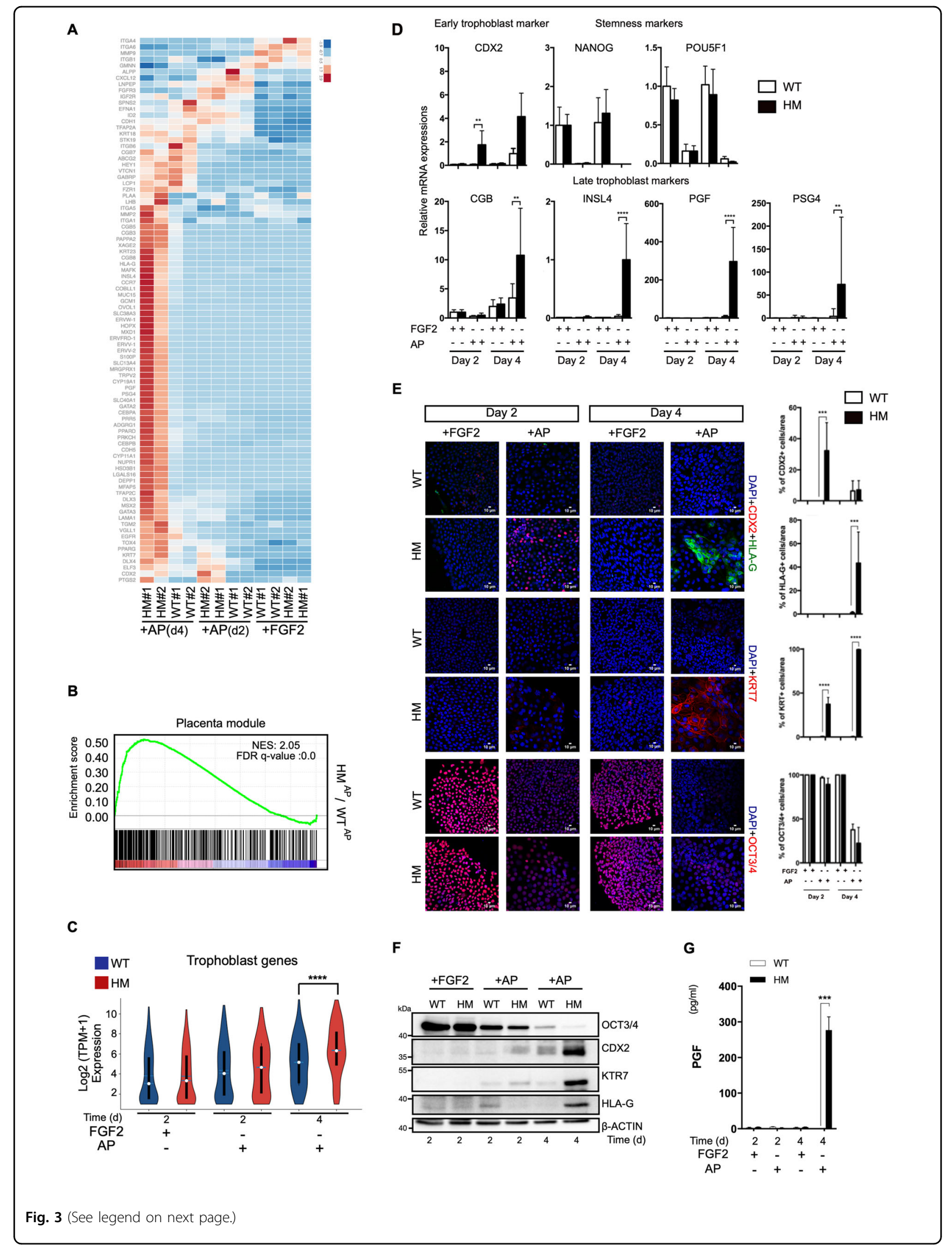


(see figure on previous page)

Fig. 3 Reduced NLRP7 expression obviates the requirement for exogenous BMP4 during trophoblast differentiation. Cells were treated with either FGF2 as a control or AP a Heatmap depicting the expression of trophoblast markers during time course of WT and HM iPSC differentiation. Color represents row-wise scaled expression using Z-score based on the raw gene expression. ( $n=2$ biological replicates). b GSEA of Placenta module. Genes were ranked according to log2 fold changes in expression comparing $\mathrm{HM}^{\mathrm{AP}}$ cells to $\mathrm{WT}^{\mathrm{AP}}$ cells on day 4. $\mathbf{c}$ Violin plot displaying logtransformed expression levels of trophoblast genes in Fig. $3 a(n=2, p$ value $<0.05$, Wilcoxon rank-sum test). $\mathbf{d}$ RT-qPCR for trophoblast and stem cell markers. e, f Immunostaining and immunoblotting for CDX2, HLA-G, KRT7, and OCT3/4. e Percentage of the cells immunostained for CDX2, HLA-G, KRT7, and OCT3/4. Scale bars; $10 \mu \mathrm{M}$. f Representative immunoblots for CDX2, HLA-G, KRT7, and OCT3/4. g 24-h PGF production as assessed by ELISA. d, $\mathbf{e}, \mathbf{g}^{*} p \leq 0.05,{ }^{* * *} p<0.005,{ }^{* * *} p<0.001$ by two-way ANOVA followed by Sidak's multiple comparison test. The bars represent mean \pm SD, $n=3$ biological replicates.

moles $^{34}$, we investigated whether increased BMP4 expression of HM cells stemmed from altered methylation status. However, we could not find a correlation between methylation status and expression levels of BMP4 (Fig. S6).

To investigate active BMP4 signaling, we generated a list of $\mathrm{BMP} 4$ responsive genes using our whole transcriptome data of BAP treatment in comparison to AP treatment of WT cells on day 2 (200 number of genes, Table S3). In $\mathrm{HM}^{\mathrm{AP}}$ cells, we observed that upregulation of this set of BMP4-responsive genes independent of BMP4 exposure (Fig. 4d). Moreover, expression of BMP4 targets, GATA-2 and GATA-3, which are also important transcription factors for trophoblast lineage commitment during embryogenesis, was significantly higher in HM cells (Fig. 4e). Western blot analysis confirmed markedly increased phosphorylation of pSMAD1/5/9 in $\mathrm{HM}^{\mathrm{AP}}$ cells during the time course of differentiation indicating an overactive BMP signaling as the underlying mechanism of augmented trophoblast differentiation of $\mathrm{HM}^{\mathrm{AP}}$ cells (Fig. 4f). Taken together, these results point to a reciprocal association between NLRP7 and BMP4 during trophoblast differentiation and suggest that hyper-trophoblast differentiation observed in HM cells with NLRP7 mutations is due to aberrant BMP4 signaling.

\section{NLRP7 reintroduction or inhibition of BMP pathway revert the excessive trophoblast differentiation of patient-specific iPSCs}

Next, we evaluated whether correction of NLRP7 expression or suppression of BMP4 signaling could restore abnormal trophoblast differentiation of HM cells. To test this, NLRP7 was stably overexpressed in HM cells (HM + NLRP7; Fig. S7a). Overexpression of NLRP7 not only led the downregulation of BMP4 and CDX2 proteins, but also restored OCT3/4 levels, indicating rescue of HM differentiation phenotypes (Fig. S7b, c).

Next, we evaluated if an inhibitor of BMP receptors ALK2 and ALK3 (LDN193189) would be able to attenuate trophoblast differentiation. LDN193189 exposed HM cells showed a similar morphological phenotype to WT cells (Fig. 5a). At the whole transcriptome level LDN193189treated cells clustered with undifferentiated iPSCs rather than AP-treated cells (Fig. S7d). Heatmap for trophoblast markers also clearly demonstrated that BMP pathway inhibition impeded the differentiation of HM cells (Fig. 5b). The global expression of trophoblast markers was also reduced in response to LDN193189, whereas pluripotency markers remained highly expressed (Fig. 5c, d). We further confirmed the dramatic decline in trophoblast gene expression upon BMP pathway inhibition by RT-qPCR (Fig. 5e).

In concordance with transcriptomic analyses, BMP pathway inhibition blocked the upregulation of CDX2, KRT7, and HLA-G and prevented the downregulation of OCT3/4 in HM cells (Fig. 5f). Overall, these results indicate that in HM cells, exit from pluripotency and rapid differentiation tendency to trophoblasts is associated with aberrant BMP signaling.

\section{Discussion}

Here, we established a patient-specific iPSC-based HM model by generating trophoblast-like cells from iPSCs carrying NLRP7 mutations via BMP4 treatment and inhibition of FGF and Activin/NODAL signaling pathways. Both WT and HM-derived trophoblast cells were able to represent characteristics of trophoblast lineage consistent with previous reports ${ }^{20,22,27,28,35,36}$. Immunostaining experiments revealed gradual acquisition of early trophoblast marker CDX2 followed by late trophoblast marker HLA-G expression during time course of differentiation in both groups. On the other hand, none of the groups were completely positive for HLA-G and presence of CDX2 positive areas on day 4 pointed to a heterogeneous differentiation stage.

The association of NLRP7 with trophoblast differentiation has been previously reported in H9 human ESCs $^{17}$ and primary trophoblasts ${ }^{18}$. Similarly, we showed expedited trophoblast differentiation in NLRP7 mutant patient-derived HM cells upon BAP exposure compared to WT cells. Like hyperplasia of trophoblast tissue in Hydatidiform moles, $\mathrm{HM}^{\mathrm{iPSC}}$ cells exhibited augmented trophoblast lineage commitment upon BAP exposure. Thus, our model recapitulates salient features of this gestational trophoblastic disease. Furthermore, these results provide an evidence of feasibility of using BMP4 derived trophoblast cells in understanding of early human 


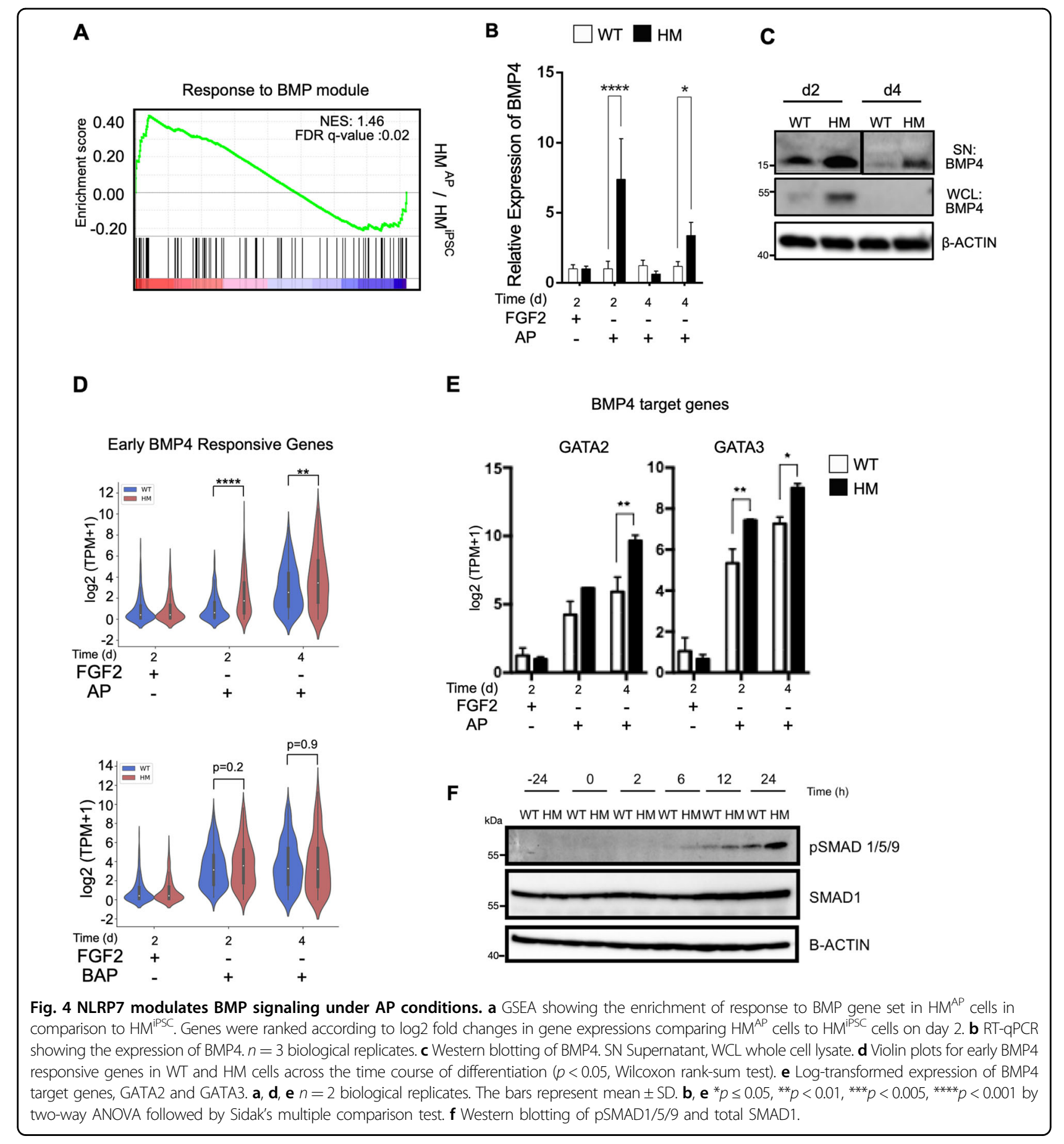

embryogenesis and placental diseases. On the other hand, while HM is characterized by hyper-proliferation of trophoblasts in vivo, we did not detect an overt proliferation phenotype in $\mathrm{HM}^{\mathrm{iPSC}}$-derived trophoblastic cells in vitro. This could be due to the absence of other factors such as the tissue microenvironment in our model or our culture conditions might not support survival of trophoblast stem cells that may drive the proliferation phenotype. Instead, we observed precocious and more extensive differentiation of the HM iPSCs to all trophoblast lineages.

We investigated the molecular mechanisms that drive cells to the trophoblast fate and uncovered an unexpected association between impaired NLRP7 and excessive BMP4 signaling. We found that $\mathrm{HM}^{\mathrm{iPSCs}}$ were still able to differentiate to trophoblasts even in the absence of exogenous BMP4, due to endogenously elevated BMP4 signaling in 


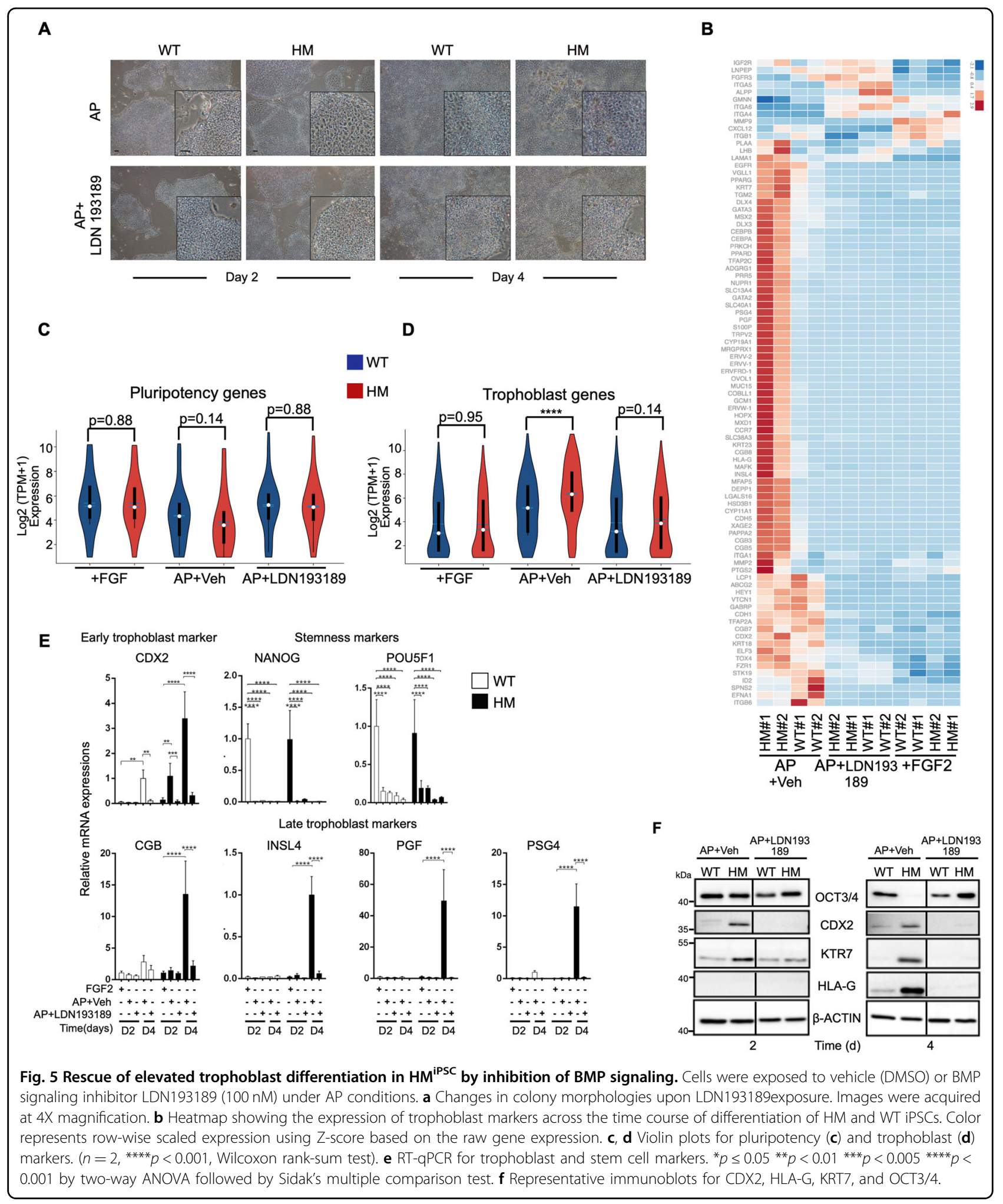

HM cells. Recently, BMP4 and GATA3 axis has been defined to be regulators of commitment to exit from pluripotency and cell fate decision in early human embryo development ${ }^{37}$. Here, we provide evidence that impaired expression of
NLRP7 is sufficient to trigger BMP4 expression, SMAD1/5/8 phosphorylation and GATA3 expression when combined with FGF and ACTIVIN/NODAL pathway inhibitors in vitro. Moreover, suppression of excessive trophoblast 
differentiation by the BMP pathway inhibitor, LDN193189, verified the involvement of BMP pathway in trophoblast lineage fate driven by impaired NLRP7 expression in HM cells. Furthermore, NLRP7 regulates the expression of other key transcription factors functioning in first cell fate decision during early embryogenesis such as CDX2.

NLRP7 has been shown to interact with the transcription factor $\mathrm{YY}^{17}$. YY1 can repress BMP signaling by inhibiting SMADs ${ }^{38,39}$ and BMP4 is known to induce CDX2 and GATA3 expressions ${ }^{40}$. YY1 is also postulated to be a member of core pluripotency network ${ }^{41}$. Hence, it is tempting to speculate that NLRP7 may restrain BMP4 levels through its interaction with YY1. NLRP7 might be indispensable for proper functioning of $\mathrm{YY} 1$ in core pluripotency network or SMAD inhibition. Although no significant differences in reprogramming efficiency were detected in HM iPSCs compared to WT iPSCs under pluripotency conditions, using exogenous FGF2 to maintain iPSCs may mask the effects caused by impaired NLRP7 expression. Since NLRP7 is a relatively novel protein with an unknown function in embryogenesis, further studies are needed to determine whether YY1NLRP7 interaction has a role in BMP4 derived abnormal trophoblast differentiation in HM.

As no embryo formation is observed in CHM, reduction in NLRP7 levels may impair ICM formation through elevated BMP4 levels by facilitating trophoblast lineage commitment or by suppressing pluripotency. Thereby, all the cells during the first cell fate decision may be directed to the trophoblast fate which results in abnormal development of placenta. In agreement with this, recent studies on in vitro preimplantation embryos have verified the requirement of NLRP7 for proper embryo development ${ }^{13,42}$. Interestingly, NLRP7 was found to be one of the most upregulated genes in embryonic carcinomas, epiblasts, and naive pluripotent stem cells along with core stem cell markers, such as OCT3/ 4 and NANOG ${ }^{43-45}$ in correlation with our results that reprogramming process itself boosts NLRP7 expression and NLRP7 mutant cells tend to lose pluripotency properties when the media is not supplemented with FGF2. We propose that during the first cell fate decision, NLRP7 is required to balance the cell fate specification between ICM and trophectoderm. While successful pregnancy requires maternal NLRP7, its deficiency is not incompatible with pluripotency under standard ESC conditions, but once the cells exit from it, differentiation to trophoblast lineage is heavily favored.

Taken together, our results show that NLRP7 mutant iPSCs can faithfully model complete hydatidiform moles. Impaired NLRP7 level predisposes pluripotent cells to the trophoblast lineage commitment by regulating the BMP4 pathway. Importantly, BMP pathway inhibition ameliorates trophoblast differentiation due to reduced NLRP7 expression, which may pave the way to therapeutic treatments for HM patients.

\section{Materials and methods}

\section{Primary culture from human skin biopsy}

The skin samples were obtained from a 30-year-old female patient and a 44-year-old healthy volunteer with informed consent under approval by Bogazici University Human Research Institutional Review Board (2014/34) by a 3 -mm punch biopsy after injection of local anesthetic. The skin samples were transferred in DMEM (GIBCO) on ice. Then, the samples were washed with PBS. After washing step, skin samples were cut into relatively small pieces by a surgical scalpel. The smaller skin samples were placed on six-well plate. Then, $22 \mathrm{~mm}$ glass cover slips were placed onto samples to stabilize them in $4{ }^{\circ} \mathrm{C}$ complete DMEM (DMEM supplemented with 10\% FBS (GIBCO), $2 \mathrm{mM} \mathrm{L-}$ Glutamine (GIBCO), 1X MEM Non-Essential Amino acids (GIBCO), $100 \mathrm{U} / \mathrm{ml}$ penicillin, and $100 \mu \mathrm{g} / \mathrm{ml}$ Streptomycin $(\mathrm{GIBCO})$ ) and incubated at $37^{\circ} \mathrm{C}$ and $5 \% \mathrm{CO}^{2}$ incubator. Medium was changed every 3-4 days. 10\% DMSO 10\% FBS $80 \%$ complete DMEM was used to freeze the cells.

\section{Patient-specific iPSC generation}

Patient-specific iPSCs were established by episomal transfection previously described elsewhere ${ }^{25,46}$. The day before the episomal transfection of reprogramming vectors, primary fibroblast cells were seeded into six-well plates at a density of $3 \times 10^{5}$ and incubated overnight at $37^{\circ} \mathrm{C}, 5 \% \mathrm{CO}^{2}$. In total, $1 \mu \mathrm{g}$ of each plasmid pCXLE-Oct3/4-shp53, pCXLE-SK, pCXLE-UL, and PCXWB- EBNA were transfected and CXLE-eGFP and pCXWB-EBNA were used as a transfection control by NeonQR Transfection System (Thermo Scientific) via electroporation with 1400 V, 20 ms, and 2pulses. Six days later from transfection, mitomycin-c (Sigma)-treated MEFs were seeded on six-well plates coated with $0.2 \%$ gelatin (Sigma). On the next day (day 7), reprogrammed cells were harvested and transferred to plates containing MEFs. Then, the medium was changed with $\mathrm{hES}$ medium containing $10 \mathrm{ng} / \mathrm{mL}$ of FGF2 (Peprotech) every other day.

\section{EBNA integration assay}

Genomic DNA from iPSCs was isolated using a commercial kit (MACHEREY-NAGEL) as indicated by the manufacturer. PCR was performed using $50 \mathrm{ng}$ genomic DNA as template per reaction with the following primers: EBNA-Fwd: AGGGCCAAGACATAGAGATG, EBNARev: GCCAATGCAACTTGGACGTT, GAPDH- Fwd: ATCACCATCTTCCAGGAGCGA, GAPDH-Rev: TTCT CCATGGTGGTGAAGACG. PCR products were sequenced by Macrogen Inc. (Korea).

\section{Teratoma formation assay}

Three confluent $10 \mathrm{~cm}$ plate were detached using regular passaging protocols and resuspended in 50\% Matrigel (Corning) and 50\% cold DMEM medium supplemented with $10 \%$ FBS, $2 \mathrm{mM}$ L-Glutamine, $100 \mathrm{U} / \mathrm{ml}$ penicillin, 
and $100 \mu \mathrm{g} / \mathrm{ml}$ streptomycin and kept on ice. The mixture was applied to three SCID mice via intramuscular injection under anesthesia. The anesthetized mice were sacrificed via an IACUC-approved method after 6-8 weeks of injection and teratoma was dissected out. Teratoma was fixed in $10 \%$ formalin. Histopathological staining and examination were performed.

\section{Trophoblast differentiation}

iPS cells were maintained routinely on MEFs with hES media $(10 \mathrm{ng} / \mathrm{mL}$ FGF2). For the trophoblast differentiation, $2.4 \times 10^{4}$ cells per square centimeter were seeded on Matrigel coated plates with conditioned hES medium by a monolayer of mitomycin-C treated MEF feeder cells (MEF-CM) containing FGF2 $(10 \mathrm{ng} / \mathrm{mL})$. On the next day, medium was changed to MEF-CM containing $4 \mathrm{ng} / \mathrm{mL}$ of FGF2. Next day, the medium was changed to BMP4 (10 ng/mL; RD Systems), the ALK4/5/7 inhibitor, A83-01 $(1 \mu \mathrm{M}$; Tocris), and the FGF2-signaling inhibitor PD173074 (0.1 $\mu$ M; Sigma) containing (BAP) hESC basal medium not conditioned with MEF feeder cells ${ }^{22,27,28}$. Control cultures were grown in the presence of FGF2 and in the absence of BAP. The medium was replenished daily.

\section{Quantitative real-time PCR}

Total RNA was extracted by Direct-zol RNA Isolation Kit (Zymogen) and cDNA was synthesized by Sensifast cDNA synthesis kit (Bioline) as described by the manufacturer. Primers were synthesized by Macrogen as listed in Supplementary Table 4. RT-qPCR was performed with SensiFAST ${ }^{\mathrm{TM}}$ SYBRQR No-ROX Kit (Bio- line) on Exicycler $^{\mathrm{TM}} 96$ (Bioneer). qRT-PCR results were analyzed by $\Delta \Delta \mathrm{Ct}$ method for relative quantifications taking GAPDH or HPRT as internal controls.

\section{Immunofluorescent staining}

The cells were fixed with $4 \%$ paraformaldehyde (PFA; Sigma) for $30 \mathrm{~min}$ at room temperature and washed with phosphate-buffered saline (PBS) three times. Then, the cells were permeabilized with $0.2 \%$ TritonX-100 (Sigma) for $20 \mathrm{~min}$ at room temperature and washed with PBS three times. The cells were blocked in 3\% BSA (Applichem) and 5\% donkey serum (Merck) in PBS for $2 \mathrm{~h}$ at RT. Immuno-labeling was performed with CDX2 (EPR2764Y, ABCAM; 1:250), KRT7 (M7018, DAKO; 1:100), OCT3/4 (sc-5279, SantaCruz; 1:100), NANOG (AB21624, ABCAM; 1:100), and mouse IgG $(0.4 \mu \mathrm{g} / \mathrm{mL})$ overnight at $+4{ }^{\circ} \mathrm{C}$. Next day, the cells were washed with $1 \mathrm{X}$ PBS three times and they were incubated with appropriate secondary antibodies conjugated with AlexaFlour 488, 555, or 568 for $3 \mathrm{~h}$ at $+4{ }^{\circ} \mathrm{C}$ in dark. The cells were washed again with $1 \mathrm{X}$ PBS for three times. Images were acquired using a confocal microscope (Leica TCS SP8, USA) and processed via ImageJ.

\section{Immunoblotting}

The cells were harvested in RIPA lysis buffer $(150 \mathrm{mM}$ $\mathrm{NaCl}, 1 \% \mathrm{NP} 40,0.5 \%$ sodium deoxycolate, $0.1 \%$ SDS, and $50 \mathrm{mM}$ Tris $\mathrm{pH}$ 7.4) supplied with protease and phosphatase inhibitors (Roche, Switzerland). For the supernatants, TCAacetone precipitation was performed. Protein samples were applied to SDS gel. Semidry transfer was performed by using Blotting papers (Sigma- Aldrich, USA) and PVDF membrane (Millipore, Ireland). After blocking with 5\% non-fat dry milk, the membranes were incubated with primary antibodies (1:1000) $+4{ }^{\circ} \mathrm{C}$ overnight. Next day, the membrane was incubated with 1:2000 HRP-coupled secondary antibodies according to primary antibody's host origin. Between all the steps, the membranes were washed with TBST for three times. Immunoblotted membranes were visualized with Syngene documentation system by using ECL HRP Substrate (Advansta, USA).

\section{Immunoassay}

PGF was measured by Human PIGF ELISA (DPG00; RD Systems) according to manufacturer's protocol. Diluted to appropriate concentration of samples and standards were transferred on 96-well plate and incubated $2 \mathrm{~h}$ at room temperature. The wells were washed four times with wash buffer and the conjugated antibody was added to the plate and incubated 2 hours at room temperature. Substrate Solution (1:1 Color Reagent A $\left(\mathrm{H}_{2} \mathrm{O}_{2}\right)$ : Color Reagent B (Tetramethylbenzidine) was incubated $30 \mathrm{~min}$ at room temperature on dark. Finally, stop Solution $\left(2 \mathrm{M} \mathrm{H}_{2} \mathrm{SO}_{4}\right)$ was added and mixed to stop the reaction and optical density was measured at 450 and $570 \mathrm{~nm}$ (VersaMax, Molecular Devices, USA).

\section{Plasmid construction and lentiviral transduction}

The pCR-Blunt-II TOPO plasmid containing the NLRP7 cDNA was purchased from Imagenes. The region that encoded for NLRP7 cDNA was PCR amplified by using SalI and NotI restriction sites added primers and ligated into pENTR1A noccDB (W48-1). Next, NLRP7 and GFP genes were subcloned into pLEX307 (Addgene 41392) lentiviral expression vector using Gateway cloning technology (Invitrogen). Sequencing procedures were performed to verify no SNP or frameshift containing plasmids. NLRP7 or GFP expressing vectors were transfected to HEK293FT cells at a density of $2.5 \times 10^{6}$ in $10 \mathrm{~cm}$ dishes to produce lentiviruses using Fugene Transfection Reagent (Promega). Virus containing media were collected for 2 days. PEG8000 (Sigma) was used to concentrate the viruses. $\mathrm{HM}^{\mathrm{iPSCs}}$ were infected with obtained viruses two times for $16 \mathrm{~h}$.

\section{RNA-sequencing analysis}

Total RNA was extracted using the Direct-zol RNA Isolation Kit (Zymogen) according to manufacturer's 
protocol ( $n=2$ in all cases). Library preparation using Truseq stranded mRNA LT Sample Prep Kit (Illumina) and RNA sequencing using Hiseq2500 (Illumina) was performed by Macrogen Inc. (Korea). RNA-seq data were processed and interpreted with the Genialis visual informatics platform (www.genialis.com). Briefly, RNA-seq reads were implemented to trimming (BBDuk), mapping (STAR), and expression quantification (featureCounts), respectively. Reads were mapped to Homo sapiens GRCh38 (Ensembl, version 92, ERCC). Differential gene expression analyses were performed using DESeq $2^{47}$. Lowly expressed genes were filtered out from the differential expression analysis input matrix. The expression level (TPM, transcripts per kilobase per million) was determined by Cufflinks (http://cufflinks.cbcb.umd.edu). Heatmaps were built with Genialis platform according to row-wise scaled expression using Z-score based on TPM values of manually curated custom gene sets of general trophoblasts, CTs, EVTs, or STs (Supplementary Tables 1 and 2). Custom gene lists were constructed from recently available literature information based on five publications ${ }^{27-29,48,49}$. Cell cycle analysis was performed using previously defined G1/S and G2/M cell cycle genes ${ }^{50}$ and heatmaps were built in $\mathrm{R}$ with pheatmap package. Benporath-ES1 gene set were used as pluripotency markers $^{51}$. The first 200 genes that are differentially upregulated in $\mathrm{WT}^{\mathrm{BAP}}$ cells relative to $\mathrm{WT}^{\mathrm{AP}}$ cells on day 2 , are assigned as early BMP4 responsive genes (Supplementary Table 3). Hierarchical clustering was performed using R, DEseq2 package to show sample distances. Violin plots were generated using Python 3.7.2 with Seaborn library. All of the RNA-seq data can be accessed with the GEO code GSE125592.

\section{Gene set enrichment analysis}

Pre-ranked differentially expressed gene lists were implemented to GSEA using default settings (http:// software.broadinstitute.org). For the identification of enrichments in pluripotency genes, BENPORATH ES1; placenta genes, Module38; BMP pathway GO response to BMP and Mitosis modules were assessed.

\section{Comparative anlaysis}

All analyses were conducted with $\mathrm{R}$ version 4.0.0. A multi-factor design was used to study the interaction effect of treatment (BAP and AP) with genotype (WT and HM) at two different time points (days 2 and 4). To determine the treatment effect for each genotype time-point combination, the genotype and time point factors were combined in a single factor and DESeq2 v1.29.4 ${ }^{47}$ was used to fit the model: counts $\sim$ combined_genotype_timepoint + treatment. Before model fitting, counts were filtered for genes with at least one count in one of the samples.
The apeglm model was used for effect size shrinkage of the $\log 2$ fold changes BAP vs AP treatment ${ }^{47,52,53}$. After shrinkage, the results were filtered for all genes with an absolute $\log$ fold change $>\log 2(1.5)$ and a Benjamini-Hochberg adjusted $p$-value of $<0.05$. The regularized logarithm was used to transform the counts of the filtered gene list for visualization (rlog DESeq2 function with BLIND = TRUE;).

To depict the transformed counts together with the estimated fold changes, the ComplexHeatmap package was used ${ }^{53}$. Prior to passing the counts to the Heatmap function, the transformed counts per gene were normalized across samples (via scale function).

\section{BMP4 methylation analysis}

Genomic DNA was extracted using Quick-DNA ${ }^{\mathrm{TM}}$ Miniprep Plus Kit (Zymo Research) according to manufacturer's protocol. Bisulfite conversion of $500 \mathrm{ng}$ DNA was conducted using EZ DNA Methylation-GoldKit ${ }^{\mathrm{TM}}$ (Zymo Research) as recommended by the manufacturer. Bisulfite modified DNA was amplified by PCR using HotStarTaq Master Mix (Qiagen). PCR and sequencing primers were designed by Pyromark Assay Design Software 2.0 (Qiagen) and available upon request. Biotinylated PCR products were mixed with streptavidin beads and converted to single strands using PyroMark Q96 Vacuum Workstation (Qiagen). Following annealing of sequencing primers, pyrosequencing was performed using Pyromark Q96 ID System (Qiagen). CpG methylation data were analyzed by PyroMark software. Samples were run in duplicate. Assays were verified using EpiTect unmethylated and methylated DNA (Qiagen).

\section{Statistics}

Statistical analyses were performed by Graphpad Prism 6.0. (San Diago, USA). The results were implemented to two-way ANOVA followed by Sidak's multiple comparison test. Wilcoxon rank-sum test was used to determine $P$ values for violin plots. The bars are presented as the mean \pm SD. $P$ values of $<0.05$ are considered statistically significant and represented as follows: ${ }^{*} P \leq 0.05$, ${ }^{* *} P<$ $0.01{ }^{* * * *} P<0.005,{ }^{* * * * * *} P<0.001$. All the experiments were conducted at least three times unless indicated otherwise.

\footnotetext{
Acknowledgements

We thank N.C.T. Emre (Bogazici University, Department of Molecular Biology and Genetics) for kindly providing pLEX307 plasmid. We are thankful to Onur Eskiocak for his help while creating most of the illustrations. We would like to thank Hannah Meyer for performing comparative analysis. We would like to thank Ahmet Kocabay and Ali Cihan Taskın for their help with mouse

experiments, Arzu Ruacan (Koç University, School of Medicine, Department of Pathology) for examination of histological sections. We would like to express gratitude to our lab member Efe Elbeyli for violin plots of RNAseq data. The authors gratefully acknowledge use of the services and facilities of the Koç University Research Center for Translational Medicine (KUTTAM), funded by the Republic of Turkey Ministry of Development. The content is solely the responsibility of the authors and does not necessarily represent the official
} 
views of the Ministry of Development. This work was supported by an EMBO Installation Grant (T.Ö.) and funded by The Scientific and Technological Research Council of Turkey (TUBITAK; projects: SBAG-112S115 and KBAG217z131; N.Ö.), Bogazici University Research Funding Office (BAP:7360; N.Ö.).

\section{Author details}

${ }^{1}$ Apoptosis and Cancer Immunology Laboratory (AKIL), Department of Molecular Biology and Genetics, Center for Life Sciences and Technologies, Bogazici University, Istanbul, Turkey. ${ }^{2}$ School of Medicine, Koç University, Istanbul, Turkey. ${ }^{3}$ Oncology Unit, Department of Obstetrics and Gynaecology, Kanuni Sultan Suleyman Training and Research Hospital, Istanbul, Turkey

\section{Conflict of interest}

The authors declare that they have no conflict of interest.

\section{Publisher's note}

Springer Nature remains neutral with regard to jurisdictional claims in published maps and institutional affiliations.

Supplementary Information accompanies this paper at (https://doi.org/ 10.1038/s41419-020-02884-1).

Received: 28 February 2020 Revised: 1 August 2020 Accepted: 3 August 2020

Published online: 19 August 2020

\section{References}

1. Li, R. \& Albertini, D. F. The road to maturation: somatic cell interaction and selforganization of the mammalian oocyte. Nat. Rev. Mol. Cell Biol. 14, 141-152 (2013).

2. Nüsslein-Volhard, C. \& Wieschaus, E. Mutations affecting segment number and polarity in Drosophila. Nature 287, 795-801 (1980).

3. Lu, X., Gao, Z., Qin, D. \& Li, L. A maternal functional module in the mammalian oocyte-to-embryo transition. Trends Mol. Med. 23, 1014-1023 (2017).

4. Nguyen, N. M. P. \& Slim, R. Genetics and epigenetics of recurrent hydatidiform moles: basic science and genetic counselling. Curr. Obstet. Gynecol. Rep. 3, 55-64 (2014)

5. Hui, P., Buza, N., Murphy, K. M. \& Ronnett, B. M. Hydatidiform moles: genetic basis and precision diagnosis. Annu. Rev. Pathol. Mech. Dis. 12, 449-485 (2017)

6. Murdoch, S. et al. Mutations in NALP7 cause recurrent hydatidiform moles and reproductive wastage in humans. Nat. Genet. 38, 300-302 (2006).

7. Milhavet, F. et al. The infevers autoinflammatory mutation online registry: update with new genes and functions. Hum. Mutat. 29, 803-808 (2008).

8. Moein-Vaziri, N. et al. Clinical and genetic-epignetic aspects of recurrent hydatidiform mole: a review of literature. Taiwan. J. Obstet. Gynecol. 57, 13-17 (2018).

9. Dixon, P. H. et al. Mutations in NLRP7 are associated with diploid biparental hydatidiform moles, but not androgenetic complete moles. J. Med. Genet. 49 206-211 (2012).

10. Soellner, L. et al. Maternal heterozygous NLRP7 variant results in recurrent reproductive failure and imprinting disturbances in the offspring. Eur. J. Hum. Genet. 25, 924-929 (2017).

11. Tong, Z.-B. et al. Mater, a maternal effect gene required for early embryonic. Nat. Genet. 26, 267-268 (2000).

12. Peng, $\mathrm{H}$. et al. Nlrp2, a maternal effect gene required for early embryonic development in the mouse. PLoS ONE 7, e30344 (2012).

13. Li, G. et al. NLRP7 is expressed in ovine ovary and associated with in vitro preimplantation embryo development. Reproduction https://doi.org/10.1530/ REP-19-0081 (2019).

14. Radian, A. D., de Almeida, L., Dorfleutner, A. \& Stehlik, C. NLRP7 and related inflammasome activating pattern recognition receptors and their function in host defense and disease. Microbes Infect. 15, 630-639 (2013).

15. Khare, S. et al. An NLRP7-containing inflammasome mediates recognition of microbial lipopeptides in human macrophages. Immunity 36, 464-476 (2012).

16. Zhou, Y. et al. Virulent mycobacterium bovis beijing strain activates the NLRP7 inflammasome in THP-1 macrophages. PLOS ONE 11, e0152853 (2016).
17. Mahadevan, S. et al. NLRP7 affects trophoblast lineage differentiation, binds to overexpressed YY1 and alters cpg methylation. Hum. Mol. Genet. 23, 706-716 (2014).

18. Abi Nahed, R. et al. NLRP7 is increased in human idiopathic fetal growth restriction and plays a critical role in trophoblast differentiation. J. Mol. Med. https://doi.org/10.1007/s00109-018-01737-x (2019).

19. Sanchez-Delgado, M. et al. Absence of maternal methylation in biparental hydatidiform moles from women with NLRP7 maternal-effect mutations reveals widespread placenta-specific imprinting. PLoS Genet. 11, e1005644 (2015).

20. Xu, R.-H. et al. BMP4 initiates human embryonic stem cell differentiation to trophoblast. Nat. Biotechnol. 20, 1261-1264 (2002).

21. Sudheer, S., Bhushan, R., Fauler, B., Lehrach, H. \& Adjaye, J. FGF inhibition directs BMP4-mediated differentiation of human embryonic stem cells to syncytiotrophoblast. Stem Cells Dev. 21, 2987-3000 (2012).

22. Amita, M. et al. Complete and unidirectional conversion of human embryonic stem cells to trophoblast by BMP4. Proc. Natl Acad. Sci. USA 110, E1212-E1221 (2013).

23. Ulker, V. et al. Novel NLRP7 mutations in familial recurrent hydatidiform mole: are NLRP7 mutations a risk for recurrent reproductive wastage? Eur. J. Obstet. Gynecol. Reprod. Biol. 170, 188-192 (2013).

24. Reddy, R. et al. The genomic architecture of NLRP7 is Alu rich and predisposes to disease-associated large deletions. Eur. J. Hum. Genet. 24, 1445-1452 (2016).

25. Okita, K. et al. An efficient nonviral method to generate integration-free human-induced pluripotent stem cells from cord blood and peripheral blood cells. Stem Cells 31, 458-466 (2013).

26. Fidan, K. et al. Generation of integration-free induced pluripotent stem cells from a patient with Familial Mediterranean Fever (FMF). Stem Cell Res. 15, 694-696 (2015)

27. Yang, $Y$. et al. Heightened potency of human pluripotent stem cell lines created by transient BMP4 exposure. Proc. Natl Acad. Sci. USA 112 E2337-E2346 (2015)

28. Yabe, S. et al. Comparison of syncytiotrophoblast generated from human embryonic stem cells and from term placentas. Proc. Natl Acad. Sci. USA 113 E2598-E2607 (2016).

29. Okae, H. et al. Derivation of human trophoblast stem cells. Cell Stem Cell 22, 50-63.e6 (2018).

30. Kubaczka, C. et al. Direct induction of trophoblast stem cells from murine fibroblasts. Cell Stem Cell 17, 557-568 (2015).

31. Isakova, G. K. \& Mead, R. A. Occurrence of amitotic division of trophoblast cell nuclei in blastocysts of the western spotted skunk (Spilogale putorius latifrons). Hereditas 140, 177-184 (2004).

32. Calvert, S. J. et al. Studies of the dynamics of nuclear clustering in human syncytiotrophoblast. Reproduction 151, 657-671 (2016).

33. Khaliq, A. et al. Localisation of placenta growth factor (PIGF) in human term placenta. Growth Factors 13, 243-250 (1996)

34. Beygo, J. et al. Deep bisulfite sequencing of aberrantly methylated loci in a patient with multiple methylation defects. PLOS ONE 8, 1-13 (2013).

35. Das, P. et al. Effects of FGF2 and oxygen in the BMP4-driven differentiation of trophoblast from human embryonic stem cells. Stem Cell Res. 1, 61-74 (2007)

36. Horii, M. et al. Human pluripotent stem cells as a model of trophoblast differentiation in both normal development and disease. Proc. Natl Acad. Sci. USA 113, E3882-E3891 (2016).

37. Gunne-Braden, A et al. GATA3 mediates a fast, irreversible commitment to BMP4-driven differentiation in human embryonic stem cells. Cell Stem Cell https://doi.org/10.1016/..stem.2020.03.005 (2020).

38. Kurisaki, K. et al. Nuclear factor $\mathrm{YY} 1$ inhibits transforming growth factor betaand bone morphogenetic protein-induced cell differentiation. Mol. Cell. Biol. 23. 4494-4510 (2003)

39. Lee, K-H., Evans, S., Ruan, T. Y. \& Lassar, A. B. SMAD-mediated modulation of YY1 activity regulates the BMP response and cardiac-specific expression of a GATA4/5/6-dependent chick Nkx2.5 enhancer. Development 131, 4709-4723 (2004).

40. Bernardo, A. S. et al. BRACHYURY and CDX2 mediate BMP-induced differentiation of human and mouse pluripotent stem cells into embryonic and extraembryonic lineages. Cell Stem Cell 9, 144-155 (2011).

41. Wang, J. et al. YY1 positively regulates transcription by targeting promoters and super-enhancers through the BAF complex in embryonic stem cells. Stem Cell Rep. https://doi.org/10.1016/j.stemcr.2018.02.004 (2018). 
42. Sills, E. S. et al. Pathogenic variant in NLRP7(19q13.42) associated with recurrent gestational trophoblastic disease: data from early embryo development observed during in vitro fertilization. Clin. Exp. Reprod. Med. 44, 40 (2017).

43. Skotheim, R. I. et al. Differentiation of human embryonal carcinomas in vitro and in vivo reveals expression profiles relevant to normal development. Cancer Res. 65, 5588-5598 (2005).

44. Qin, H. et al. YAP induces human naive pluripotency. Cell Rep. 14, 2301-2312 (2016).

45. Kilens, S. et al. Parallel derivation of isogenic human primed and naive induced pluripotent stem cells. Nat. Commun. 9, 360 (2018).

46. Fidan, K., Ebrahimi, A., Çağlayan, Ö. H., Özçimen, B. \& Önder, T. T. Transgene-free Disease-specific iPSC Generation From Fibroblasts And Peripheral Blood Mononuclear Cells. 215-231 (Humana Press, New York, NY, 2015).

47. Love, M. I., Huber, W. \& Anders, S. Moderated estimation of fold change and dispersion for RNA-seq data with DESeq2. Genome Biol. 15, 550 (2014).
48. Lee, C. Q. E. et al. What is trophoblast? A combination of criteria define human first-trimester trophoblast. Stem Cell Rep. 6, 257-272 (2016).

49. Vento-Tormo, R. et al. Single-cell reconstruction of the early maternal-fetal interface in humans. Nature 563, 347-353 (2018).

50. Tirosh, l. et al. Dissecting the multicellular ecosystem of metastatic melanoma by single-cell RNA-seq. Science. https://doi.org/10.1126/science.aad0501 (2016).

51. Ben-Porath, I. et al. An embryonic stem cell-like gene expression signature in poorly differentiated aggressive human tumors. Nat. Genet. 40, 499-507 (2008).

52. Zhu, A., Ibrahim, J. G. \& Love, M. I. Heavy-Tailed prior distributions for sequence count data: removing the noise and preserving large differences. Bioinformatics https://doi.org/10.1093/bioinformatics/bty895 (2019).

53. Gu, Z., Eils, R. \& Schlesner, M. Complex heatmaps reveal patterns and correlations in multidimensional genomic data. Bioinformatics https://doi.org/ 10.1093/bioinformatics/btw313 (2016) 